\title{
1 Characteristics of the Long Duration Pulses in a Shunt Linear Voltage Regulator ${ }^{\text {th }}$
}

2

4

\section{Abstract}

Shunt linear voltage regulators are still used in situations where other kinds of regulators are not advised. This paper explores a mechanism liable to induce long duration pulses $(\sim 100 \mu \mathrm{s})$ in these devices, which is eventually demonstrated using a pulsed laser facility. Data issued from these tests helps to understand how the electrical network parameters as well as the non-idealities of the devices affect the characteristics of the transients. Finally, this phenomenon is investigated in similar structures with identical purpose.

6 Keywords: Laser tests, long duration pulses, peak detector effect, power electronics, single event transients, shunt 7 voltage regulators

8 PACS: 07.50.Ek, 07.89.+b, 42.62.-b, 61.82.Fk, 84.30.Jc, 85.40.-e

${ }^{a}$ Departamento de Física Aplicada III, Facultad de Físicas, Universidad Complutense de Madrid (UCM), 28040 Madrid (Spain)
${ }^{b}$ Centro de Láseres Ultrarrápidos, Facultad de Químicas, Universidad Complutense de Madrid (UCM), 28040 Madrid (Spain)

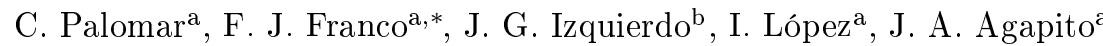

\section{Introduction}

The family of linear voltage regulators (or simply references, if the device is not designed to provide a high output current) is classically divided into two large subfamilies: Series and shunt regulators. The second family, on which this work focuses, are devices that set an accurate voltage value in a specific node of the circuit working in parallel with the load. It is recommended for low power systems, also if the power supply is higher than $40 \mathrm{~V}$, and, finally, is useful when negative, limiting or floating references are required [1]. A typical example is the Zener diode (Fig. 1a). The Zener diode sets the output near its breakdown voltage, $V_{Z}$. Thus, the power supply provides a constant quiescent current, $I_{S} \approx \frac{V_{C C}-V_{Z}}{R_{S}}$, independent of the load characteristics. The excess of current, $I_{P}=I_{S}-I_{O}>0$, is drained by the diode. However, in case of needing an accurate value of the output voltage, the simple Zener diode must be discarded in benefit of structures such as that shown in Fig. 1b. In this case, an operational amplifier (op amp) sets the output voltage to $(1+k) \cdot V_{R E F}$. Typically, $V_{R E F}$ is the output of a band-gap cell, a Zener diode, etc. Another function of the op amp is biasing the base of a PNP bipolar transistor to drain the excess of current. A PMOS transistor can replace the PNP one [2] as well as an NPN or NMOS transistor [3]. In this case, the op amp inputs must swap their roles to avoid positive feedback.

Voltage regulators have been tested in radiation environments investigating the effects of the accumulated damage or single event effects, as a recent review paper has summarised [4], to be used in harsh environments such as space or accelerators $[5,6,7]$. In particular, shunt linear voltage regulators have been tested in radiation facilities,

This work was supported in part by the MCINN projects AYA2009-13300-C03-03 and Consolider SAUUL CSD2007-00013, by MCINN Grant CTQ2008- 02578/BQU, and by UCM-BSCH.

${ }^{*}$ Corresponding author

Email address: fjfranco@fis.ucm.es (F. J. Franco ) 


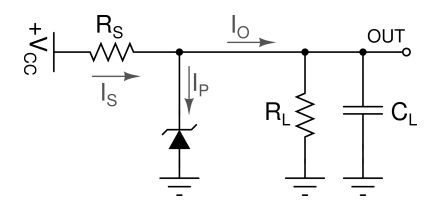

(a)

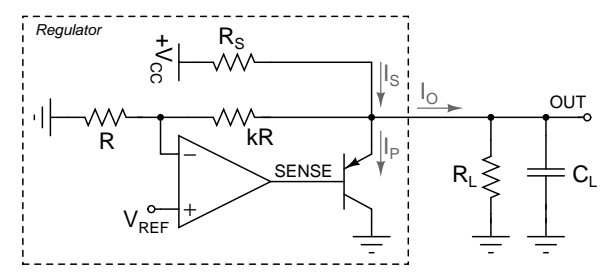

(b)

Figure 1: Several kinds of shunt linear voltage regulators: A simple Zener diode (a) and a more accurate version based on an op amp (b).

sometimes focusing on total ionizing dose $[8,9,10,11]$, sometimes on the single event transients (SETs) in these devices [3, 12, 13]. A recent paper [3] has investigated the characteristics of the SETs in a COTS (Commercialoff the shelf) shunt regulator, the LM4050, and successfully associated the transient duration with the dynamical parameters. Unlike the series linear voltage regulators, the influence of the external configuration devices on the transients of shunt regulators has not deeply studied. A common practice is to add a bypass capacitor to the output of the voltage regulator $\left(C_{L}\right.$ in Fig. 1a-b) with several purposes: removal of high-frequency noise, stabilization of the circuits, reservoir of current, etc. Other papers have shown that, contrary to the first impression, this extra device can make the transients more dangerous in series linear regulators $[14,15]$. In shunt regulators, long duration pulses (LDPs) might occur due to a similar mechanism: Let us suppose that, due to the impact of an energetic heavy ion, a negative transient appears at the output of the op amp, called SENSE in Fig. 1b. As the transistor is in common-collector configuration, the output voltage, $V_{O}$ tries to follow the evolution of $V_{S N S}$ so a negative peak occurs at the regulator output. Then, the op amp quickly reacts since $V_{-}=\frac{V_{O}(t)}{1+k}<V_{+}=V_{R E F}$, quitting the linear zone and jumping to positive saturation.

If the capacitor were not present, the transient would vanish after a few microseconds since, in its trip to the positive saturation state, the output of the op amp grows so much that $V_{-}>V_{+}$. Negative feedback acts so the output voltage starts decreasing and softly returns to the stable DC value. However, the presence of the capacitor, which was efficiently discharged by the transistor but can be charged again only by $I_{S}$, freezes the output voltage so the op amp eventually reaches the positive saturation state. Then, the transistor stays cut off and the circuit behaves like a classical RC network. Fig. 2 shows the expected behaviour of the transients according a SPICE simulation and explains why these transients are so long and intrinsically bipolar. Also, characteristic times and voltages are defined therein. This transient was simulated injecting $3 \mathrm{pC}$ in a transistor, called Q09, inside the op amp gain stage. More details about the simulation can be found in Section 3 .

This phenomenon is just a modification of the "peak detector effect", observed in series linear voltage regulators and references $[14,15]$. Some characteristics of the transients can be easily deduced. First of all, when the pass transistor goes to OFF state, the network becomes a typical RC network (From $\mathbf{C}$ to $\mathbf{E}$ in Fig. 2). Calling 


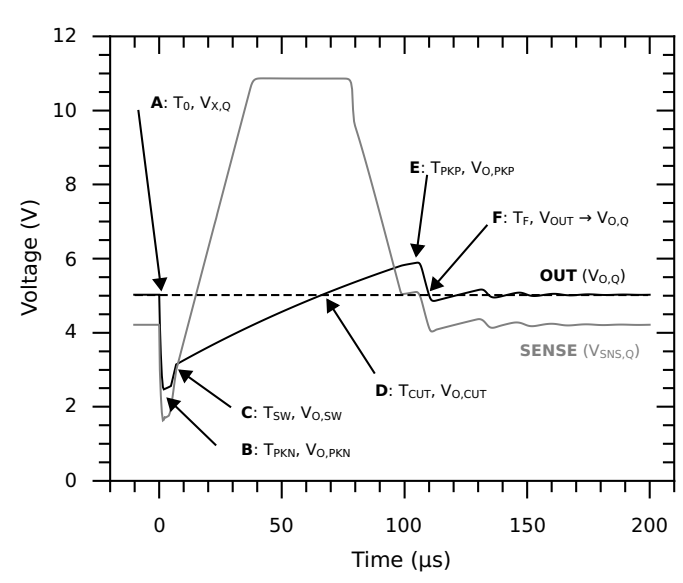

Figure 2: Predicted behaviour of a long duration pulse in shunt voltage regulators. After the transient trigger (A), the OUT \& SENSE voltages reaches the minimum (B). However, due to the fact that $V_{+}-V_{-}>0$, the SENSE voltage, $V_{S N S}$, goes to the positive saturation voltage switching off the transistor in such a way that the capacitor recovery becomes that of a typical RC network (C). Much later, the output voltage goes beyond the DC value (D), $V_{+}-V_{-}<0$ so the op amp returns to the linear zone. Meanwhile, the output voltage goes on increasing and reaches a top peak value (E) before returning to the DC output voltage (F).

$V_{O, S W} \equiv V_{O}\left(t=T_{S W}\right)(\mathbf{C}$ in Fig. 2), the final value would be

$$
V_{O}(t \rightarrow \infty)=V_{\infty}=\frac{R_{L}^{*}}{R_{L}^{*}+R_{S}} \cdot V_{C C},
$$

52 $\quad R_{L}^{*}$ being $R_{L} / /[(1+k) \cdot R]$. Then, the temporal evolution is:

$$
V_{O}(t)=V_{\infty}+\left[V_{O, S W}-V_{\infty}\right] \cdot \exp \left(-\frac{t-T_{S W}}{\tau}\right)
$$

53 with

$$
\tau=\left(R_{L}^{*} / / R_{S}\right) \cdot C_{L}
$$

${ }_{54}$ From now on, $\tau$ will be called "theoretical discharge time". Finally, another interesting characteristic of the transients 55 is the size of the negative peak, $V_{O, P K N}$. A simple way to determine the parameters that affect the value of this parameter is the following. Once the transient starts, the external PNP/PMOS transistor is set on a low-impedance state. Let us suppose that the transistor collector/drain current is limited to $I_{P, M A X}$ due to non-ideal effects such as collector/drain resistance, base resistance, high current injection, etc. If the transient reaches its lowest value at $t=T_{P K N}$ and provided that the transistor is discharging a capacitor, it is very easy to deduce that

$$
\left|V_{O, P K N}-V_{O, Q}\right| \approx C_{L}^{-1} \cdot T_{P K N} \cdot I_{P, M A X}
$$

60

61

62

63

64

65

66

In other words, the negative peak transient should be inversely proportional to the capacitor value. A similar conclusion, using more sophisticated models, was deduced and experimentally observed in series linear regulators [15].

\section{Experimental set-up}

In order to verify that the hypothetical LDPs actually occur in shunt linear voltage regulators, the network in Fig. $1 \mathrm{~b}$ was built using an LM124A, the behaviour of which is well known, as its core. This amplifier was fed back with an 2N2907A PNP transistor and resistors of $100 \& 33 \mathrm{k} \Omega($ DC Gain $\approx 4)$. The rest of parameters were variable 
Table 1: Top and bottom values of the circuit parameters.

\begin{tabular}{|c|cc|}
\hline Parameter & Bottom & Top \\
\hline$V_{R E F}$ & $0.75 \mathrm{~V}$ & $1.50 \mathrm{~V}$ \\
$V_{O}$ & $3.0 \mathrm{~V}$ & $6.0 \mathrm{~V}$ \\
$V_{C C}$ & $10 \mathrm{~V}$ & $16 \mathrm{~V}$ \\
\hline$R_{S}$ & $220 \Omega$ & $390 \Omega$ \\
$R_{L}$ & $680 \Omega$ & $2.7 \mathrm{k} \Omega$ \\
$C_{L}$ & $220 \mathrm{nF}$ & $4.7 \mu \mathrm{F}$ \\
\hline
\end{tabular}

(Table 1). In the second round of experiments, the PNP transistor was replaced by a BS250, a silicon DMOS-PFET for high-speed switching applications. Unlike typical integrated shunt regulators, the op amp was biased by the power supply and not by its own output. Otherwise, the structure would not have worked.

Heavy-ion facilities have been traditionally the place where electronic devices are tested for SET sensitivity. However, it is widely accepted that a pulsed laser is a good tool to test bipolar technologies since the elements are much bigger than the typical spot size [16]. The possible LDPs associated with the shunt voltage regulators were investigated at the UCM laser facility [17]. In this facility, a 60-fs pulsed laser was used to induce SETs in the op amp. Its wavelength was set to $800 \mathrm{~nm}$ (spot size $\sim 1 \mu \mathrm{m}$ ) and the energy to $60 \mathrm{pJ}$ (absorption coefficient, $\sim 0.085 \mu^{-1}$, penetration depth, $\left.\sim 11.8 \mu \mathrm{m}[16]\right)$. It is interesting to correlate this energy with the equivalent LET of an ion. In 2000, a work by McMorrow [18] calculated that, in many not very integrated technologies and with single photon absorption, $1 \mathrm{pJ} \sim 3 \mathrm{MeV} \cdot \mathrm{mg} \cdot \mathrm{cm}^{-2}$. This ratio has been confirmed by more recent works [14]. In conclusion, the equivalent laser LET is in the order of $180 \mathrm{MeV} \cdot \mathrm{mg} \cdot \mathrm{cm}^{-2}$. This value is not realistic but offers the chance to obtain repeatable transients on the oscilloscope screen. Let us bear in mind that the main purpose of the paper is to investigate how external devices affect the characteristics of the transients. Therefore, a repeatable transient is mandatory to draw any conclusion.

The LM124A, in ceramic package, was mechanically decapsulated to reach the internal components. Namely, the laser was focused on the collector-base junction of a transistor inside the common-collector / common-emitter gain stage. This 60- $\mu \mathrm{m}$ wide vertical NPN transistor is called Q12 in the manufacturer's datasheet but Q09 in some scientific works $[15,19,20,21]$. Transients originating here are sudden negative peaks.

The main circuit signals $\left(V_{O U T}, V_{S N S}, V_{C C}\right)$ were captured with a Yokogawa DLM6000 oscilloscope triggered by a logic signal coming from the laser system. These analogue signals were numerically treated with an $11^{\text {th }}$ order Savitzky-Golay filter to remove quantization and high-frequency noise. Every time that the signals were recorded, the oscilloscope stored 2500 samples of each, of which 250 were taken before the laser arrival. These subset of samples were used to compute the mean DC values of those signals, $V_{O, Q}, V_{S N S, Q} \& V_{C C, Q}$. Besides, the error of every signal was calculated as the maximum of $a$ ) the instrumental error of the oscilloscope $( \pm 20 \mathrm{mV})$ and $b) 1.98$ times the standard deviation of the subsets of 250 samples, (Confidence of 95\% in Gaussian distributions). The error margin of any parameter derived from these signals is calculated from these original values $\left(\Delta V_{O}, \Delta V_{S N S}\right.$ $\left.\& \Delta V_{C C}\right)$ according to the theory of propagation of uncertainty. Every signal was measured 5 times to verify the consistence of the measurements. 


\section{Simulations in SPICE}

LDPs were also investigated using SPICE simulations. The LM124A was simulated with a SPICE micromodel developed with the following three assumptions: First, the internal topology was that shown in a paper by Savage et al. [19]; second, the SPICE transistors parameters were obtained from state-of-the-art tables, such as those found in [22]; Third, the area of the transistor inside the bias mirrors were scaled to obtain the currents shown by the manufacturer in the LM124A datasheet.

Transients are emulated using piecewise current sources with a duration in the order of $1 \mathrm{ps}$, much smaller than the device response time. This micromodel has several drawbacks. For instance, there is not any difference between vertical and lateral transistors. Besides, as the sizes of the transistors are not those of the actual device, the injected charge should not be extrapolated from simulations to actual transients. However, the micromodel is accurate enough to predict the way in which the feedback network or loads affect the shape of the SETs. Indeed, it has been successfully used by the authors in the study of SETs in structures containing an LM124A [15, 20].

Finally, the SPICE engine was the NGSpice v. 25, a GNU fork ${ }^{1}$ of the original Berkeley Spice 3f5. Fig. 2, which illustrates the LDP parameters, was obtained with this SPICE micromodel.

\section{Results}

First of all, there was not any significant difference between the behaviour of the PNP and the PMOS versions. In both cases, the shape of the OUT signal was similar to that Fig. 2. Minor differences are in the shape of $S N S$ signal without influence on the shape of $O U T$ at any rate. Therefore, redundant information is removed and only results on the PNP version will be reported unless otherwise noted.

The first step in this work is to verify that the expected LDPs do occur. An example is Fig. 3a-c, which were registered using a $2 \mathrm{~N} 2907 \mathrm{~A}$ as pass transistor with different values of $C_{L}$ and other passive components shown in the caption. One can see that the shape of the transients reminds of those predicted in Fig. 2. Inside the graphs, the supposed-to-be exponential zone was fitted to a function with a final value of $7.59 \mathrm{~V}$, predicted by Eq. 1 with the parameters in the caption of Fig. 3. From these fits, one can extract the value of the experimental characteristic time, $\tau_{E X P}$, which must be equal to $\tau$, obtained from Eq. 3 . These procedure was repeated in all of the hundreds of measurements recorded at the laser facility. Sometimes, an anomalously large value of error appears due to one of these two reasons: First, the spontaneous appearance of oscillations, with an amplitude of 50-150 mV, superposed to the DC value (E. g., 3a). Second, the error increases if the exponential zone is too flat (E. g., Fig. 3c). Both values of $\tau$ are compared in Fig. 4 and the results of the linear fit shown in Table 2. Parameters corresponding to the linear fit of the 4.7- $\mu \mathrm{F}$ capacitor have wide error margins due to the worse quality of the original data, calculated from exponential curves like that of 3c. Ideally, the predicted slope in the linear fits is 1 and the only divergence should be attributed to the tolerance of the discrete devices. Resistor can be easily measured with the 4-wire technique to refine the calculation of $\tau$ so any remaining divergence must be related to the capacitor. According to the manufacturers, the tolerance of these devices is $\pm 10-20 \%$, too small to explain some values of B in Table 2 .

\footnotetext{
${ }^{1}$ http://ngspice.sourceforge.net/
} 
Table 2: Results of fitting Fig. 4 to $\tau_{E X P}=A+B \cdot \tau . r$ is the Pearson's correlation coefficient.

\begin{tabular}{|c|c|c|c|}
\hline Sample & $\mathrm{A}$ & $\mathrm{B}$ & $r^{2}$ \\
\hline \hline $220 \mathrm{nF}$ & $-0.3 \pm 0.3$ & $1.04 \pm 0.02$ & 0.999 \\
\hline $470 \mathrm{nF}$ & $-1.9 \pm 0.7$ & $0.88 \pm 0.02$ & 0.999 \\
\hline $1.0 \mu \mathrm{F}$ & $-3.4 \pm 1.6$ & $0.87 \pm 0.02$ & 0.999 \\
\hline $2.2 \mu \mathrm{F}$ & $-18 \pm 10$ & $0.90 \pm 0.02$ & 0.996 \\
\hline $4.7 \mu \mathrm{F}$ & $-18 \pm 34$ & $0.69 \pm 0.06$ & 0.964 \\
\hline
\end{tabular}

Table 3: $V_{P K N}$ vs. $V_{O, Q}\left(V_{C C}=12 \mathrm{~V}, C_{L}=1 \mu \mathrm{F}, R_{S}=389 \Omega, R_{L}^{*}=672 \Omega\right)$

\begin{tabular}{|c|c|}
\hline$V_{O, Q}$ & $V_{O, Q}-V_{P K N}$ \\
\hline $2.94 \pm 0.21 \mathrm{~V}$ & $0.47 \pm 0.29 \mathrm{~V}$ \\
$3.98 \pm 0.05 \mathrm{~V}$ & $1.24 \pm 0.13 \mathrm{~V}$ \\
$4.95 \pm 0.07 \mathrm{~V}$ & $1.40 \pm 0.13 \mathrm{~V}$ \\
$5.94 \pm 0.02 \mathrm{~V}$ & $1.41 \pm 0.04 \mathrm{~V}$ \\
\hline
\end{tabular}

This phenomenon will be discussed in Section 5.1. Now, other characteristics such as the negative \& positive peaks and the transient duration will be studied.

\subsection{Negative peak}

According to Eq. 4, the size of the negative peak, $\left|V_{O, P K N}-V_{O, Q}\right|$ is proportional to $C_{L}^{-1}$. Fig. 5 shows that the negative peak of actual transients seem to follow a hyperbolic trend with the exceptions of the $2.2-\mu \mathrm{F}$ and, more clearly, the $4.7-\mu \mathrm{F}$ capacitors. Just like the strange behaviour during the discharge interval, this anomaly will be explained in the discussion regarding the non-idealities of the capacitor. Another important conclusion to be drawn from this graph is that dots corresponding to different combinations of $R_{L} \& R_{S}$ are pretty close to each other. Therefore, the deppendence of $V_{O, P K N}$ on these parameters, if any, is negligible compared to that on $C_{L}$.

Apart from the capacitor value, the only parameter that the negative peak voltage depends on is the DC output voltage. In fact, the negative peak voltage decreases with $V_{O, Q}$ (Table 3). In the case of the PMOS transistors, transients were even absent if $V_{O, Q} \leq 4.0 \mathrm{~V}$. However, behind this behaviour there is a very simple reason: The op amp output is too close to the negative saturation voltage and has no margin to fall down. Therefore, the capacitor cannot be efficiently drained and the negative peak voltage is smaller. A simple reconnection of the op amp negative power supply from ground to $-5 \mathrm{~V}$ makes the transients with $V_{O, Q}=3 \mathrm{~V}$ as large as those with $V_{O, Q}=6 \mathrm{~V}$.

\subsection{Positive peak size}

A simple way of estimating the size of the positive peak consists in accepting that higher values of $\frac{d V_{O}}{d t}$ at $t=T_{D}$ lead to higher values of $V_{O, P K P}$. Differentiating Eq. 2 and taking into account that $V_{O}\left(T_{D}\right)=V_{\infty}+\left[V_{O, S W}-V_{\infty}\right]$. $\exp \left(\frac{T_{D}-T_{S W}}{\tau}\right)=V_{O, Q}$, it is easy to demonstrate that

$$
\left.\frac{d V_{O}}{d t}\right|_{t=T_{D}}=\frac{V_{\infty}-V_{O, Q}}{\tau}
$$

149 In other words, positive peaks increase with $\tau^{-1}=\frac{1}{C_{L} \cdot\left(R_{S} / / R_{L}^{*}\right)}$ and $\frac{R_{L}^{*}}{R_{L}^{*}+R_{S}} V_{C C}-V_{O, Q}$. Fig. 6 demonstrates that both parameters affect the size of the positive peak as predicted by this simple approach. 


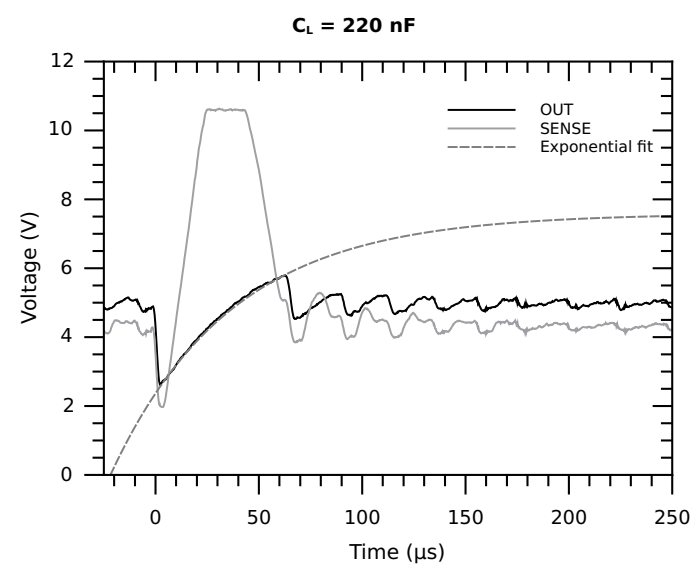

(a)

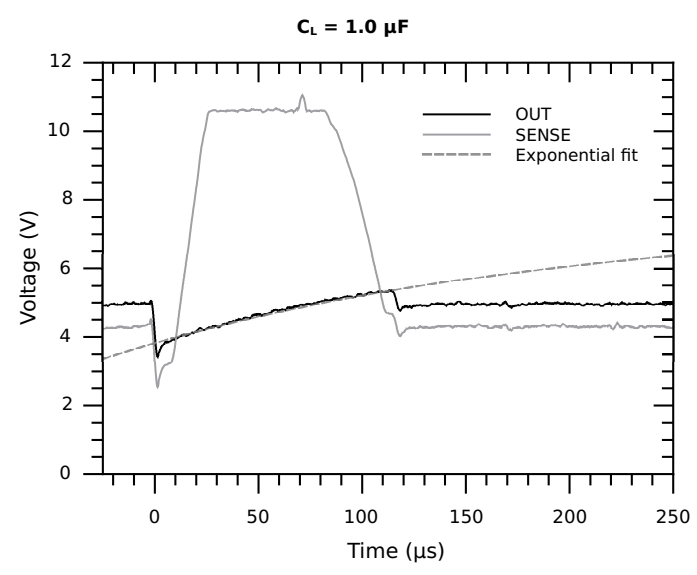

(b)

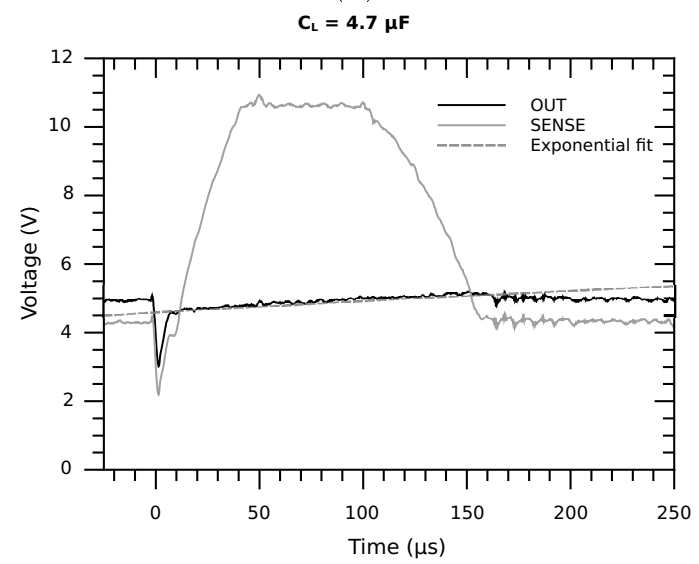

(c)

Figure 3: Experimental measurements of long duration pulses with $V_{O}=5 \mathrm{~V}, V_{C C}=12 \mathrm{~V}, R_{S}=389 \Omega$, and $R_{L}^{*}=672 \Omega$. The dashed line is the exponential fit of the transient while the transistor is cut off. 


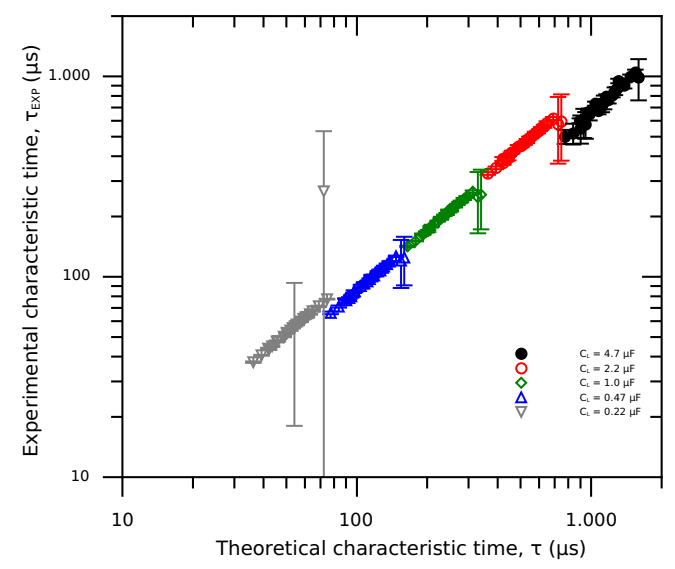

Figure 4: Experimental vs. theoretical values of $\tau$ obtained from actual measurements. Each color is associated with a capacitor value. Every set of data is obtained varying $R_{L} \& R_{S}$ so there are 25 dots in each set. The largest error bars are related to small oscillations or to very flat exponential stages. Axis are in logarithm format in order to show the whole population of dots so the graph is slightly distorted.

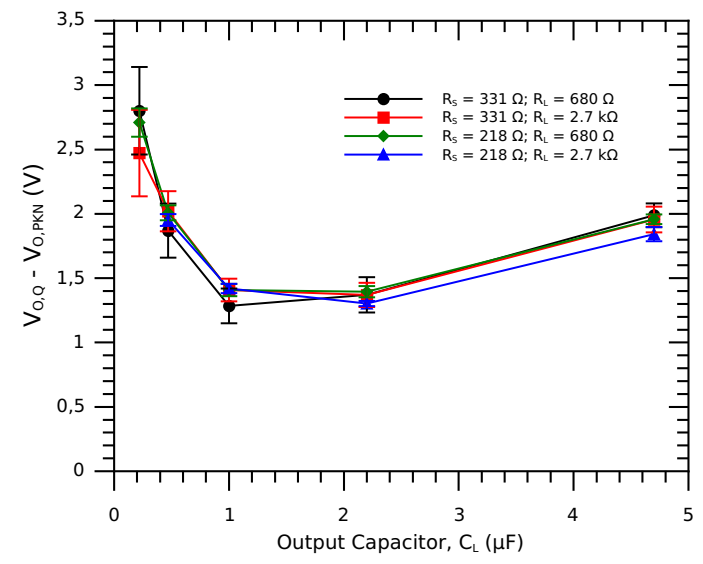

Figure 5: Evolution of the negative peak voltage in proportion to the output capacitor value with different values of network resistors in actual measurements $\left(V_{C C}=12 \mathrm{~V}, V_{O}=5 \mathrm{~V}\right)$. 


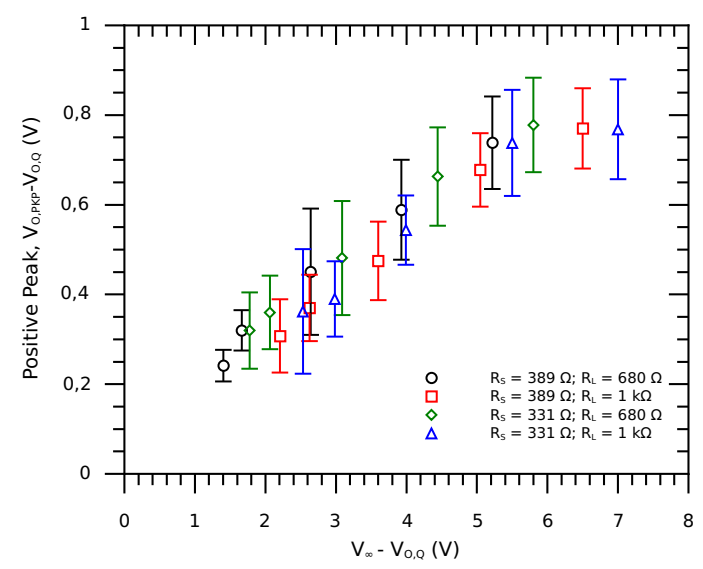

(a)

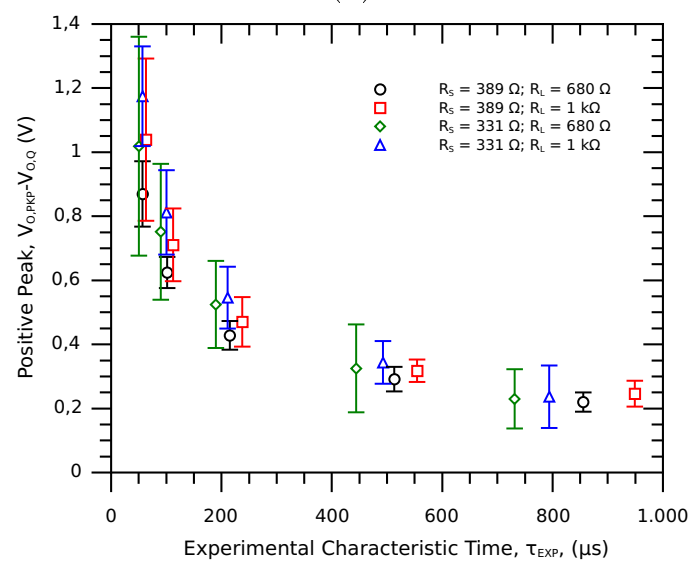

(b)

Figure 6: Dependence of $V_{O, P K P}$ on the parameters on the electrical network in actual measurements. In the first graph (a), $V_{O}=5 V$, $10 \mathrm{~V}<V_{C C}<16 \mathrm{~V}$ and $C_{L}=1 \mu \mathrm{F}$. In the second one (b), the experimental characteristic time changes with $C_{L}$ and $R_{S} \& R_{L}$ $\left(V_{O}=5 \mathrm{~V}, V_{C C}=12 \mathrm{~V}\right)$. 


\subsection{Transient duration}

The most significant characteristic of the long duration pulses is that they are much longer than other SETs. The definition of duration in these transients is difficult. Sometimes, the transient does not fall smoothly to the DC value but shows a sort of damped oscillation. Therefore, for the sake of simplicity, the transient duration was defined as the first time that the output signal crosses the DC output voltage after the positive peak (F in Fig. 2). Recorded transients lasted for 40-160 $\mu$ s and, in general, the duration increases with $\tau$ (Fig. 3).

\section{Discussion}

In the previous section, it was demonstrated that long duration pulses can occur in shunt voltage regulators. This phenomenon is intrinsically related to the electric topology so it should be expected not only in those regulators built with discrete devices but those built in integrated circuits. However, there are some points such as the characteristic time anomaly, the strange behaviour of the negative pulse, etc., that must be carefully studied. Besides, it is interesting to determine if the LDPs also occur in other topologies of shunt voltage regulators.

\subsection{Non-idealities of the capacitors and pass transistors}

In the experiments, commercial capacitors were used to investigate the long duration pulses. The smallest capacitor $(220 \mathrm{nF})$ was made in polyester and the rest of devices were Al-electrolytic capacitors. These are extremely non-linear and dependent on the frequency [23]. Their characteristics were measured with an Agilent 4294A LCR impedance meter (Fig. 7) and are very useful to explain the odd characteristic of some transients.

First of all, let us remember that the Laplace transform of Eq. 2 is

$$
V_{O}(s)=\frac{V_{\infty}}{s}+\frac{V_{O, S W}-V_{\infty}}{s+\tau^{-1}}
$$

An exponential phase factor related to the delay, $T_{S W}$, has been removed for the sake of clarity. Eq. 6 offers two readings: a) There is a peak at $s=0$ that quickly vanishes as $s \rightarrow \infty$. b) the frequency spectrum is almost constant and not negligible for any component below $s \sim \tau^{-1}$. Typically, $\tau \sim 0.04-1 \mathrm{~ms}$ in most of the transients (Fig. 4) so it is sensible to use the capacitance value on the order of $1-10 \mathrm{kHz}$ instead of using the $\mathrm{DC}$ nominal value to refine the calculation of $\tau_{E X P} . C_{L}$ values in this frequency range (Fig. 7a) are much closer than the nominal ones to those values issued from the experimental discharge time of the $\mathrm{RC}$ network (Table 2).

In the laboratory, it was determined that the highest collector current of the $2 \mathrm{~N} 2907$ sample was $\sim \tilde{6} 00 \mathrm{~mA}$. Besides, we observed that, typically, the negative peak was reached $1.8 \pm 0.7 \mu$ s after the beginning of the SET. Therefore, according to Eq. $4,\left|V_{O, P K N}-V_{O, Q}\right|$ must be between the hyperbolae $0.66 \cdot 10^{-6} \cdot C_{L}^{-1} \& 1.5 \cdot 10^{-6} \cdot C_{L}^{-1}$. In Fig. 5, samples corresponding to $C_{L}=0.22,0.47 \& 1.0 \mu \mathrm{F}$ follow this prediction but those related to the larger values show peaks much larger than expected. Fig. 7b helps to explain this anomaly. Both capacitors are also characterized by having a high ESR value. Recently, Allen et al. discovered the influence of the capacitor ESR value on the size of the transients in series voltage regulators [24]. Basically, the higher value of ESR at high frequency, the larger the size of the transients. Therefore, a similar behaviour might be expected in shunt voltage regulators.

Fig. 8 shows the simulation of the negative transient after injecting $5 \mathrm{pC}$ in Q09 of the LM124-like SPICE micromodel. This simulation confirms the previous assumption since, in the inset of the graph, one can see that the 


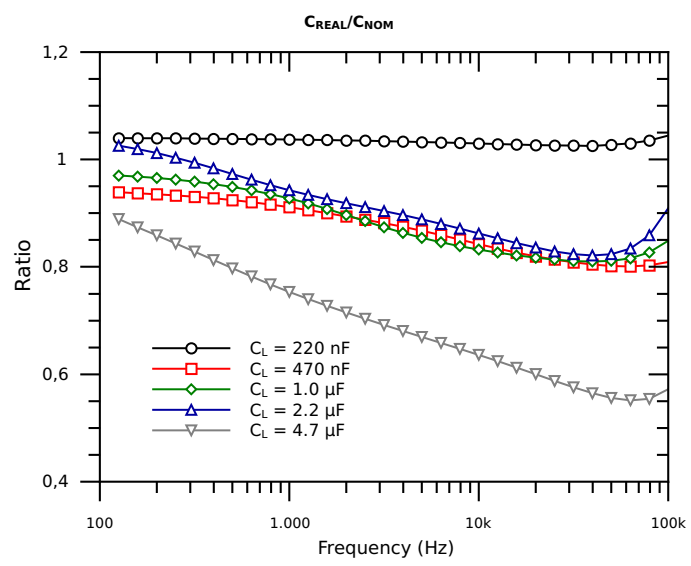

(a)

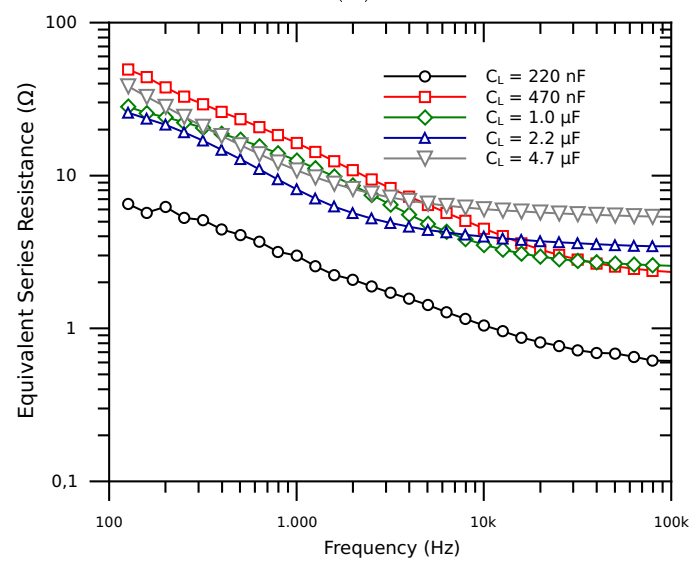

(b)

Figure 7: Characteristics of the actual capacitors as a function of the work frequency. The normalized capacitance (a) and the equivalent series resistance, ESR (b).

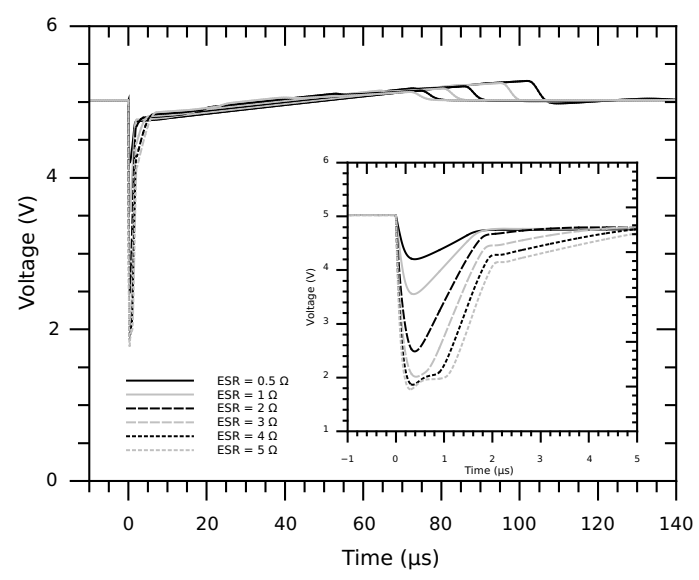

Figure 8: SPICE simulation of the effects of ESR in the shape of the transients $\left(C_{L}=4.7 \mu F, R_{L}=1 k \Omega, R_{S}=220 \Omega, V_{C C}=12 V\right.$, $V_{O}=5 \mathrm{~V}$ ). 


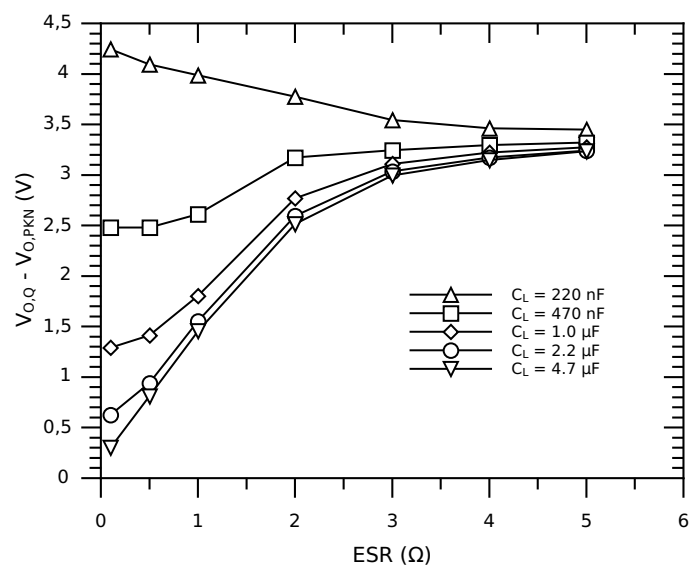

(a)

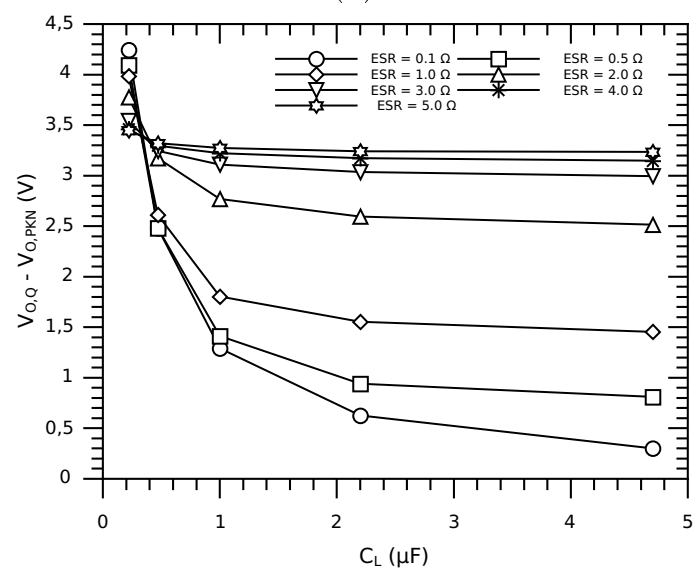

(b)

Figure 9: Evolution of the negative peak voltage with the ESR value for different values of $C_{L}$ in SPICE simulations (a). In the second graph, $C_{L}$ is used as X-variable and ERS as parameter $\left(R_{L}=1 k \Omega, R_{S}=220 \Omega, V_{C C}=12 \mathrm{~V}, V_{O}=5 \mathrm{~V}\right)$.

peak voltage grows very quickly with the ESR value. This behaviour is summarised in Fig. 9a-b. In the case of the largest capacitors, an ESR value in the order of $5 \Omega$ makes the voltage several times higher than expected with an ideal capacitor. Another conclusion of Fig. $9 \mathrm{~b}$ is that, for the lowest value of ESR, the values of $\left|V_{O, P K N}-V_{O, Q}\right|$ are distributed following a hyperbola accounting for the predictions of Eq. 4. Finally, it is important to remark that the influence of ESR on $\left|V_{O, P K N}-V_{O, Q}\right|$ is identical to the one reported by Allen et al. in series voltage regulators [24]. In other words, it does not matter if the regulator belongs to the shunt or series families.

Besides, an interesting property of the simulated transients can be observed in Fig. 8b: The ESR value has influence on $V_{O, S W}$. In this simulation, one can realise that the value of $\left|V_{O, Q}-V_{O, S W}\right|$ falls as the ESR value increases. However, this is not a general trend since, in other simulation, the behaviour is just the contrary. Further work is necessary in this research line since the value of $V_{O, S W}$ determines the size and duration of the LDP.

As the ESR is a parameter that shifts as the capacitor ages [25], these results also make one think that transients in voltage regulator designed to work for a long time in radiation environments will change as years go by due to the ESR drift. 


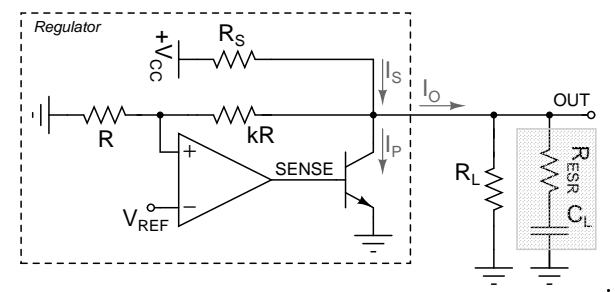

Figure 10: Shunt voltage regulator with an NPN as pass transistor. The parasitic ESR has been added to the output capacitor.

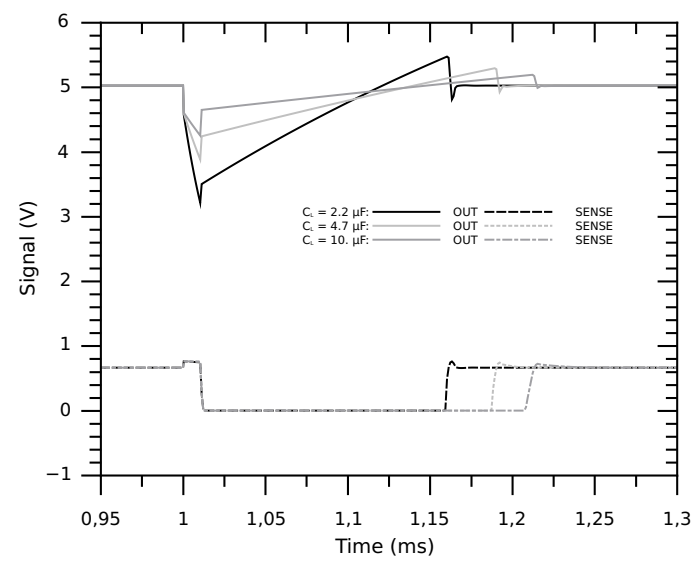

Figure 11: SPICE simulation of the structure of Fig. 10 with an $2 \mathrm{~N} 2222 \mathrm{~A}, R_{L}=1 \mathrm{k} \Omega, R_{S}=220 \Omega$, and different values of the output capacitor. An ESR of $1 \Omega$ was added to stabilise the system.

\subsection{Other topologies}

The structure shown in Fig. 1 is only one of the possible topologies to build shunt voltage regulators. However, there are other structures that can be also used to design blocks with similar features. A choice is replacing the PNP (PMOS) by an NPN (NMOS) transistor and changing the feedback loop to stabilize the device (Fig. 10). Apparently, this topology is sensitive to positive peaks at the op amp output, SENSE. A positive peak activates the NPN BE-junction leading to a sudden increase of $I_{P}$, which discharges the capacitor. In consequence, $V_{O U T} \& V_{+}$ decrease and the op amp reacts going to negative saturation, cutting off the pass transistor. Thus, the capacitor can be charged only with the help of $I_{S}$, just like the structure of Fig. 1 .

Unfortunately, this structure could not be tested in the laser facility since despite the fact of trying several devices, the regulator based on an LM124A was unstable. Fortunately, SPICE simulations were stable and the phenomenon could be investigated (Fig. 11). In this case, single events were simulated injecting charge in QR1, an open-base NPN transistor working as a resistor in the gain stage of the LM124A [19, 20]. As expected, the LDPs occur and the shape and other characteristics of the output voltage are similar to those of the PNP version of the shunt voltage regulator.

Another point to deal with is the fact that, in integrated circuits, the positive power supply of the op amps is usually connected to the regulator output. Thus, the shunt voltage regulator becomes a two-terminal device. Let us bear in mind that, in our experiments, the positive power supply was independent of the output of the device. This strategy is not unusual and has been explored by other authors [13]. In the structure of Fig. 10, this detail lacks importance as the op amp output falls down to the other power supply, ground. In the case of the PNP regulator, 


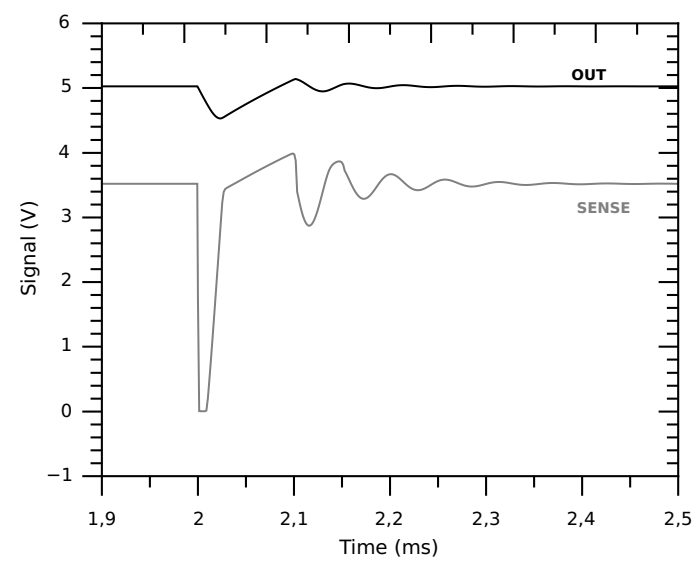

Figure 12: SPICE simulation of the structure of Fig. 1 with a PMOS transistor and the op amp biased by the output itself. Parameters concerning the simulation: $V_{C C}=24 \mathrm{~V}, R_{L}=10 \mathrm{k} \Omega, R_{S}=2.2 \mathrm{k} \Omega, C_{L}=0.47 \mu F, E S R=1 \Omega$. The PMOS transistor model, in a 350-nm technology, was downloaded from http://www.mosis.org and simulated with $W=0.35 \mu \mathrm{m}$ and $L=350 \mu \mathrm{m}$.

a problem appears: the LM124A is not rail-to-rail so the output voltage cannot be large enough to cut off the PNP transistor in Fig. 1. On the contrary, the PMOS solution does not share this drawback and the LM124A can switch off the PMOS transistor. Fig. 12 corresponds to a SPICE simulation of the structure, demonstrating that LDPs can occur even with the op amp biased by the regulator output. Curiously, simulations show that transients are usually longer for this configuration. This is due to the fact that, in many op amps, the frequency response worsens as the power supply value decreases [26], slowing down the op amp recovery.

\section{Conclusion}

In this paper, long duration pulses related to the presence of external output capacitors have been found in shunt voltage regulators. These pulses were observed in SPICE simulations and in laser tests and can be expected if the regulator is exposed to heavy-ion radiation. In spite of the fact that the experiments were performed in blocks built with discrete devices, this family of single event transients is intrinsically related to the topology of the electrical network so they can occur in integrated regulators.

These transients are always bipolar: A sharp negative spike, the size of which strongly depends not only on the value of the output capacitor but also on other parameters such as the equivalent series resistance at high frequency. Later, there is a positive peak the size of which is mostly related to the values of the passive discrete devices such as the capacitor, load \& source resistors, etc. In between, there is an interval during which the capacitor is slowly recharged. The duration of this stage depends not only on the values of the network resistors and output capacitor but also on the value of the equivalent series resistance of the capacitor, which affects the voltage at which the exponential decay starts. In general, the duration of the transient is on the order of $0.1 \mathrm{~ms}$ and, more or less, can be related to the value of the characteristic time of the circuit, $\tau$.

\section{Acknoledgements}

The authors thank Dr. Enrique San Andrés, from the Departamento de Física Aplicada III, Facultad de Físicas, Universidad Complutense de Madrid (UCM), for his help and support at characterising the frequency behaviour of the capacitors. 


\section{References}

[1] Series or Shunt Voltage Reference?. Maxim Integrated Application Notes. On-line at http://www.maximintegrated.com/an4003, Tech. rep. (Mar. 2007).

[2] S. Rioux, A. Lacourse, Y. Savaria, M. Meunier, Design methods for CMOS low-current finely tunable voltage references covering a wide output range, in: IEEE International Symposium on Circuits and Systems (ISCAS), 2005, pp. 4257-4260 Vol. 5. doi:10.1109/ISCAS.2005.1465571.

[3] N. J.-H. Roche, S. Buchner, L. Dusseau, K. Kruckmeyer, J. Boch, J. Warner, F. Saigne, D. McMorrow, G. Auriel, B. Azais, Correlation of Dynamic Parameter Modification and ASET Sensitivity in a Shunt Voltage Reference, IEEE Transactions on Nuclear Science 59 (6) (2012) 2756-2763. doi:10.1109/TNS.2012.2224127.

[4] P. Adell, L. Scheick, Radiation Effects in Power Systems: A Review, IEEE Transactions on Nuclear Science 60 (3) (2013) 1929-1952. doi:10.1109/TNS.2013.2262235.

[5] A. Drozhdin, M. Huhtinen, N. Mokhov, Accelerator related background in the CMS detector at LHC, Nuclear Instruments and Methods in Physics Research Section A: Accelerators, Spectrometers, Detectors and Associated Equipment 381 (2-3) (1996) 531-544. doi:10.1016/S0168-9002(96)00807-8.

[6] S. Duzellier, Radiation effects on electronic devices in space, Aerospace Science and Technology 9 (1) (2005) 93-99. doi:10.1016/j.ast.2004.08.006.

[7] M. Bagatin, A. Coniglio, M. D’Arienzo, A. De Lorenzi, S. Gerardin, A. Paccagnella, R. Pasqualotto, S. Peruzzo, S. Sandri, Radiation Environment in the ITER Neutral Beam Injector Prototype, IEEE Transactions on Nuclear Science 59 (4) (2012) 1099-1104. doi:10.1109/TNS.2012.2187461.

[8] W. Abare, F. Brueggeman, R. Pease, J. Krieg, M. Simons, Comparative analysis of low dose-rate, accelerated, and standard cobalt-60 radiation response data for a low-dropout voltage regulator and a voltage reference, in: IEEE Radiation Effects Data Workshop (REDW), 2002, pp. 177-180. doi:10.1109/REDW.2002.1045550.

[9] J. Hatch, LM185 voltage reference radiation tests: Variable temperature and bias conditions, in: 12th European Conference on Radiation and Its Effects on Components and Systems (RADECS), 2011, pp. 931-933. doi:10.1109/RADECS.2011.6131332.

[10] K. Kruckmeyer, T. Trinh, L. McGee, A. Kelly, Impact of Reference Voltage on the ELDRS Characteristics of the LM4050 Shunt Voltage Reference, in: IEEE Radiation Effects Data Workshop (REDW), 2011, pp. 1-5. doi:10.1109/REDW.2010.6062532.

[11] S. McClure, J. Gorelick, R. Pease, B. Rax, R. Ladbury, Total dose performance of radiation hardened voltage regulators and references, in: IEEE Radiation Effects Data Workshop (REDW), 2001, pp. 1-5. doi:10.1109/REDW.2001.960440.

[12] P. Adell, R. Schrimpf, C. Cirba, W. Holman, X. Zhu, H. Barnaby, O. Mion, Single event transient effects in a voltage reference, Microelectronics Reliability 45 (2) (2005) 355-359. doi:10.1016/j.microrel.2004.05.029. 
[13] A. Kelly, P. Adell, A. F. Witulski, W. Holman, R. Schrimpf, V. Pouget, Total Dose and Single Event Transients in Linear Voltage Regulators, IEEE Transactions on Nuclear Science 54 (4) (2007) 1327-1334. doi:10.1109/TNS.2007.903243.

[14] A. Zanchi, S. Buchner, C. Hafer, S. Hisano, D. Kerwin, Investigation and Mitigation of Analog SET on a Bandgap Reference in Triple-Well CMOS Using Pulsed Laser Techniques, IEEE Transactions on Nuclear Science 58 (6) (2011) 2570-2577. doi:10.1109/TNS.2011.2172460.

[15] C. Palomar, F. J. Franco, I. Lopez-Calle, J. G. Izquierdo, J. A. Agapito, Peak Detector Effect in Low-Dropout Regulators, IEEE Transactions on Nuclear Science 60 (4) (2013) 2666-2674. doi:10.1109/TNS.2012.2232305.

[16] S. Buchner, F. Miller, V. Pouget, D. McMorrow, Pulsed-Laser Testing for Single-Event Effects Investigations, IEEE Transactions on Nuclear Science 60 (3) (2013) 1852-1875. doi:10.1109/TNS.2013.2255312.

[17] I. Lopez-Calle, F. Franco, J. Agapito, J. G. Izquierdo, P. Reviriego, J. Maestro, TPA laser source for SEE test at UCM, in: 12th European Conference on Radiation and Its Effects on Components and Systems (RADECS), 2011, pp. 454-457. doi:10.1109/RADECS.2011.6131421.

[18] D. McMorrow, J. Melinger, S. Buchner, T. Scott, R. Brown, N. Haddad, Application of a pulsed laser for evaluation and optimization of SEU-hard designs [CMOS], IEEE Transactions on Nuclear Science 47 (3) (2000) 559-565. doi:10.1109/23.856480.

[19] M. Savage, T. Turflinger, J. Titus, H. Barsun, A. Sternberg, Y. Boulghassoul, L. Massengill, Variations in SET pulse shapes in the LM124A and LM111, in: IEEE Radiation Effects Data Workshop (REDW), 2003, pp. 121-126. doi:10.1109/REDW.2003.1281361.

[20] F. J. Franco, I. Lopez-Calle, J. G. Izquierdo, J. A. Agapito, Modification of the LM124 Single Event Transients by Load Resistors, IEEE Transactions on Nuclear Science 57 (1) (2010) 358-365. doi:10.1109/TNS.2009.2037894.

[21] F. Roig, L. Dusseau, A. Khachatrian, N. J.-H. Roche, A. Privat, J.-R. Vaillé, J. Boch, J. Warner, F. Saigne, S. Buchner, D. McMorrow, P. Ribeiro, G. Auriel, B. Azais, R. Marec, P. Calvel, P. Bezerra, R. Ecoffet, Modeling and Investigations on TID-ASETs Synergistic Effect in LM124 Operational Amplifier From Three Different Manufacturers, IEEE Transactions on Nuclear Science 60 (6) (2013) In Press.

[22] P. R. Gray, P. J. Hurst, S. H. Lewis, R. G. Meyer, Analysis and Design of Analog Integrated Circuits, 5th Edition, John Wileys and Sons, Inc., 2010.

[23] A. Amaral, A. J. M. Cardoso, An Experimental Technique for Estimating the ESR and Reactance Intrinsic Values of Aluminum Electrolytic Capacitors, in: Proceedings of the IEEE Instrumentation and Measurement Technology Conference (IMTC), 2006, pp. 1820-1825. doi:10.1109/IMTC.2006.328273.

[24] G. Allen, P. Adell, D. Chen, P. Musil, Single-Event Transient Testing of Low Dropout PNP Series Linear Voltage Regulators, IEEE Transactions on Nuclear Science 59 (6) (2012) 2764-2771. doi:10.1109/TNS.2012.2222442. 
307

308

309

310

311

312

[25] A. Lahyani, P. Venet, G. Grellet, P.-J. Viverge, Failure prediction of electrolytic capacitors during operation of a switchmode power supply, IEEE Transactions on Power Electronics 13 (6) (1998) 1199-1207. doi:10.1109/63.728347.

[26] F. J. Franco, Y. Zong, J. A. Agapito, New Details About the Frequency Behavior of Irradiated Bipolar Operational Amplifiers, IEEE Transactions on Nuclear Science 53 (4) (2006) 1931-1938. doi:10.1109/TNS.2006.880948. 